\title{
Artrose medial do joelho: Uma patologia de evolução progressiva
}

\section{Medial Knee Arthrosis: A Pathology with a Progressive Evolution}

\author{
Gilberto Luis Camanho ${ }^{1}$ () \\ ${ }^{1}$ Departamento de Ortopedia e Traumatologia, Faculdade de \\ Medicina, Universidade de São Paulo, São Paulo, SP, Brasil \\ Rev Bras Ortop 2021;56(2):133-137.
}

\author{
Endereço para correspondência Gilberto Luis Camanho, \\ Departamento de Ortopedia e Traumatologia, Faculdade de Medicina, \\ Universidade de São Paulo, Rua Dr. Ovídio Pires de Campos, 333, \\ Cerqueira Cesar, São Paulo, SP, 05403-010, Brasil \\ (e-mail: gilbertocamanho@gmail.com).
}

\section{Resumo \\ Palavras-chave \\ - artrose \\ - menisco/lesões \\ - osteonecrose}

A artrose medial do joelho é uma patologia evolutiva que ocorre em decorrência de desequilíbrio muscular progressivo. Os músculos da região do joelho têm um grande desequilíbrio, provocado pela diferença de potência e braço de alavanca. Com a progressão da vida, este desequilíbrio se manifesta de forma mais importante, especialmente em decorrência da perda de força muscular em função do envelhecimento. Posturas patológicas passam a ocorrer e determinar zonas de apoio e pressão lesivas para a articulação. A lesão meniscal é típica na evolução desta patologia, assim como a lesão da cartilagem. O reconhecimento desta patologia possibilita resultados bons com tratamentos menos agressivos, como a correção do desequilíbrio muscular e consequente reeducação do apoio da articulação. A meniscectomia econômica e parcial traz bons resultados nas fases iniciais do processo degenerativo. A evolução progressiva leva à degeneração do joelho e à consequente necessidade de cirurgias mais amplas.

Medial arthrosis of the knee is an evolutionary pathology that occurs due to progressive muscle imbalance. The muscles of the knee region have a large imbalance caused by the difference of power and lever arm. With the progression of life, this imbalance manifests itself more importantly, especially due to the loss of muscle strength due to aging. Pathological postures begin to occur and determine areas of support and pressure harmful to the joint. Meniscal injury is typical in the evolution of this pathology, as well as cartilage injury. The recognition of this pathology enables good results with less aggressive treatments, such as correction of muscle imbalance and consequent reeducation of joint support. Economic and partial meniscectomy brings good results in the early stages of the degenerative process. Progressive evolution leads to knee degeneration and the consequent need for broader surgeries. recebido

06 de Janeiro de 2020

aceito

20 de Fevereiro de 2020

Publicado online

Julho 22, 2020
DOI https://doi.org/

10.1055/s-0040-1710333. ISSN 0102-3616. (c) 2020. Sociedade Brasileira de Ortopedia e Traumatologia. All rights reserved.

This is an open access article published by Thieme under the terms of the Creative Commons Attribution-NonDerivative-NonCommercial-License, permitting copying and reproduction so long as the original work is given appropriate credit. Contents may not be used for commercial purposes, or adapted, remixed, transformed or built upon. (https://creativecommons.org/ licenses/by-nc-nd/4.0/)

Thieme Revinter Publicações Ltda., Rua do Matoso 170, Rio de Janeiro, RJ, CEP 20270-135, Brazil 


\section{Introdução}

A artrose é uma condição clinica frequente, com a qual geralmente lidamos sem uma sistematização adequada.

Consideram-se três tipos de artrose:

1. artrose de causa inflamatória: aquela decorrente do processo degenerativo inflamatório, a osteoartrite, ou consequente a artrites inflamatórias ou infecciosas, na qual a lesão do osso subcondral é a mais importante;

2. artrose pós-traumática: aquela consequente a traumas que afetem a superfície articular, como fraturas, osteocondrites, na qual a cartilagem é a mais afetada;

3. artrose mecânica: aquela decorrente de desvios de eixo ou instabilidades articulares, na qual o osso subcondral e a cartilagem são afetados.

Trata-se de condições diferentes que, se confundidas, levarão a atitudes terapêuticas desastrosas.

No joelho, e provavelmente em outras articulações, como no ombro, há um tipo de artrose que decorre da evolução de um desequilíbrio muscular e que leva a uma artrose do tipo mecânica, com características muito claras; no joelho, é a artrose que compromete o compartimento medial, determinando uma deformidade em varo. Se não for tratado, é um processo degenerativo que evolui progressivamente. Analisaremos a evolução deste processo degenerativo de causa mecânica.

\section{Etiopatogenia}

O joelho é submetido a forças musculares mal equilibradas, que, pela atividade e hipertrofia, se equilibram.

Tem um único grupo extensor e três grupos flexores, sendo dois, além de flexores, rotadores, um medial e outro lateral. Os flexores mediais são os músculos isquiotibiais, o lateral é o bíceps femoral, e os gêmeos são potentes flexores.

O equilíbrio destas forças permite movimentos harmônicos nas atividades do dia a dia e no esporte.

Observamos, na clínica diária, casos de desequilíbrio muscular nos quais o grupo extensor (quadríceps) supera o flexor (bíceps, semitendíneo, grácil e sartório, e os gêmeos), ou em que os flexores superam o quadríceps, levando a diversas queixas.

Com o passar dos anos, perdemos de $30 \%$ a $40 \%$ da força muscular, e esta perda não é uniforme: os músculos maiores sofrerão mais do que os menores.

O quadríceps perde parte de sua força, e os flexores, embora também percam, prevalecem; como os flexores mediais são em maior número, ocorre progressivamente uma deformidade em flexão e rotação interna, que leva ao varo.

Em um estudo em portadores de varismo degenerativo versus voluntários normais, Molina et al $^{1}$ observaram que chegam a ocorrer 9 graus de rotação interna da tíbia no varo patológico da artrose medial.

A falência do ligamento cruzado anterior (LCA) é progressiva, e foi descrita com clareza por $A_{h l b a ̈ c k}{ }^{2}$ na evolução da artrose medial do joelho. No seu clássico trabalho, o autor limita a indicação da osteotomia valgizante da tíbia a joelhos "estáveis," ou seja, com LCA ainda continente; e, para tal, define os limites de competência do LCA.
A deformidade em varo ocorre progressivamente, e determina um aumento de tensão no complexo ligamentar lateral, que se opõe à deformidade em varo. Este desequilíbrio postural sobrecarrega o LCA, posicionado para evitar o valgo, que entra progressivamente em falência, possibilitando a anteriorização da tíbia em relação ao fêmur.

Esta mudança de apoio e de zonas de pressão provocadas pela "migração" tibial determina lesões meniscais e de cartilagem de vários graus, provocando no compartimento medial uma artrose característica.

Intra-articularmente, o menisco é a primeira estrutura a sofrer, pois recebe um aumento posteromedial de apoio na transição entre o corpo e o corno posterior.

Ocorre uma migração anterior da tíbia em relação ao fêmur ( - Figura 1).

A lesão meniscal desta artrose medial é típica na transição já descrita, e optamos por chamá-la de lesão meniscal por fadiga, pelo seu aspecto progressivo. A cartilagem e o osso subcondral podem apresentar lesões pela mesma razão.

Consideraremos isoladamente as características de cada uma destas lesões; porém, elas ocorrem concomitantemente, podendo haver lesão meniscal com graus variáveis de lesões do osso subcondral e da cartilagem, ou qualquer outra associação de lesões.

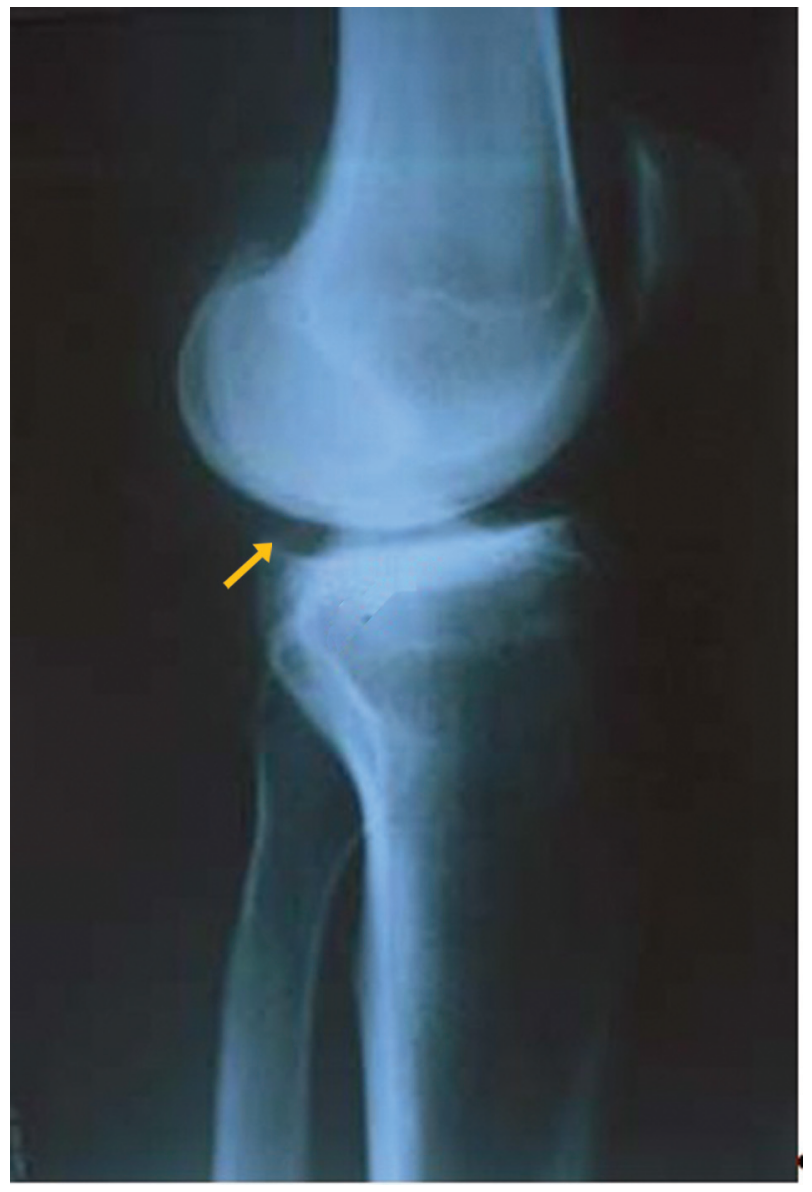

Fig. 1 Radiografia em perfil do joelho demonstrando anteriorizaçãoda tíbia em relação ao fêmur. Nota-se o osteófito posterior na tíbia, característico desta migração. 


\section{Lesão Meniscal por Fadiga}

O comprometimento da estrutura do menisco pode ocorrer por diversas razões: desde traumas até processos degenerativos podem alterar a sua integridade. O quadro clínico é muito semelhante independente da etiologia, mas a abordagem terapêutica é completamente diferente dependendo da etiologia da lesão.

Em 2006, Camanho et al $^{3}$ estudaram um grupo de pacientes portadores de lesão meniscal isolada diagnosticada por ressonância magnética (RM), que foram divididos em três grupos segundo a etiologia:

1. um grupo com lesão claramente traumática;

2. um grupo com lesão meniscal claramente decorrente do processo degenerativo da articulação; e

3. um grupo que foi chamado"portador de lesão meniscal por fadiga", no qual a lesão meniscal tinha características próprias, diferentes das dos dois outros grupos;

Chamou atenção dos pesquisadores a semelhança quanto ao perfil dos pacientes do grupo portador de lesão degenerativa e aquele portador da lesão que se chamou de lesão por fadiga. Eram pacientes na mesma faixa etária, com uma mesma distribuição segundo o sexo, mas quadro clínico e radiológico diverso e abordagem terapêutica diferente.

Nos pacientes com lesão meniscal degenerativa, o comprometimento do menisco é parte do processo degenerativo da articulação, fazendo da lesão meniscal uma parte do problema, nem sempre a responsável pelos sintomas principais.

Nos pacientes portadores de lesão meniscal por fadiga, as características clinicas são bastante claras, e a lesão meniscal tem características próprias. São elas:

1. dor de início espontâneo ou após pequeno trauma (desproporcional ao sintoma); ${ }^{4}$

2. dor noturna que exige mudança de posição do joelho;

3. exame radiográfico normal, sem sinais de artrose; e

4. ressonância magnética demonstrando lesão radial na transição entre o corpo e o corno posterior do menisco medial com extrusão do corpo meniscal, sem sinais de artrose (-Figura 2).

A grande diferença é o comprometimento da cartilagem, que ocorre na evolução da artrose medial.

\section{Lesão Osteocondral}

Pelo apoio medial e posterior, à instabilidade decorrente da insuficiência do LCA, e a deformidade em varo à superfície articular sofre, e ocorrem lesões osteocondrais progressivamente maiores.

A extensão destas lesões e a deformidade em varo definem um divisor na gravidade da artrose medial.

Inicialmente, são lesões focais na região posteromedial que progridem à medida que a instabilidade gerada pela falência do LCA e o varismo se acentuam.

A progressão da deformidade e da anteriorização levam a um aumento da área da lesão condral, agravando o quadro.

$O$ varismo acentuado e a anteriorização da tíbia definem a gravidade do quadro.

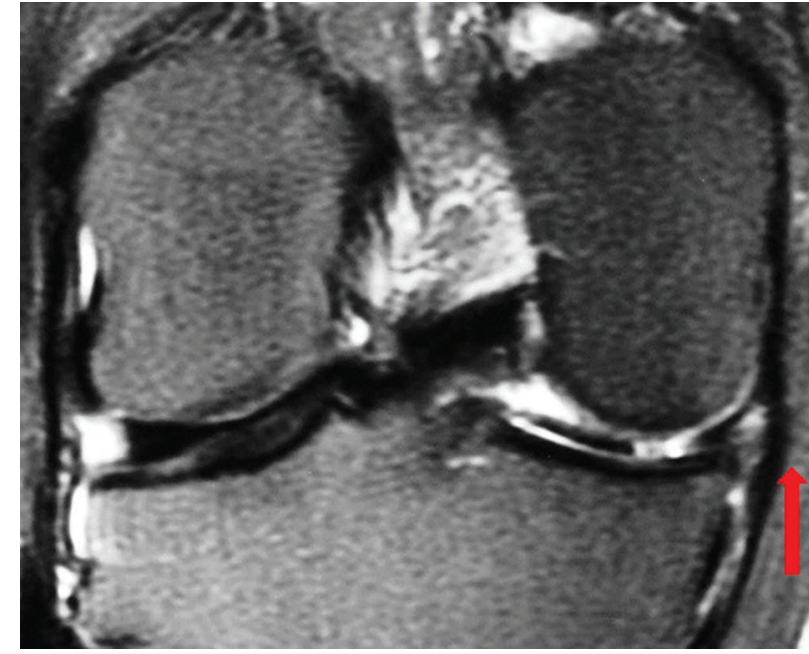

Fig. 2 Imagem de ressonância magnética(RM) do joelho demonstrando lesão meniscalposteromedial.

A sobrecarga do osso subcondral pode levar a uma fratura por fadiga, que já foi confundida com osteonecrose primária. ${ }^{5}$ A chamada osteonecrose primária é uma lesão que ocorre em decorrência de fratura por insuficiência, como demonstraram Yamamoto e Bullough. ${ }^{6}$

O quadro clínico é muito semelhante ao da lesão meniscal destes pacientes: dor súbita após um esforço ou um pequeno trauma. A imagem de edema ósseo na RM é característica (-Figura 3).

\section{Quadro Clínico}

O quadro clínico é progressivo, e depende das lesões intrínsecas. Em geral, inicia-se por uma dor aguda e importante, sem causa proporcional ao sintoma, ${ }^{4}$ na região medial do joelho. O exame físico demonstra a posição em flexão e

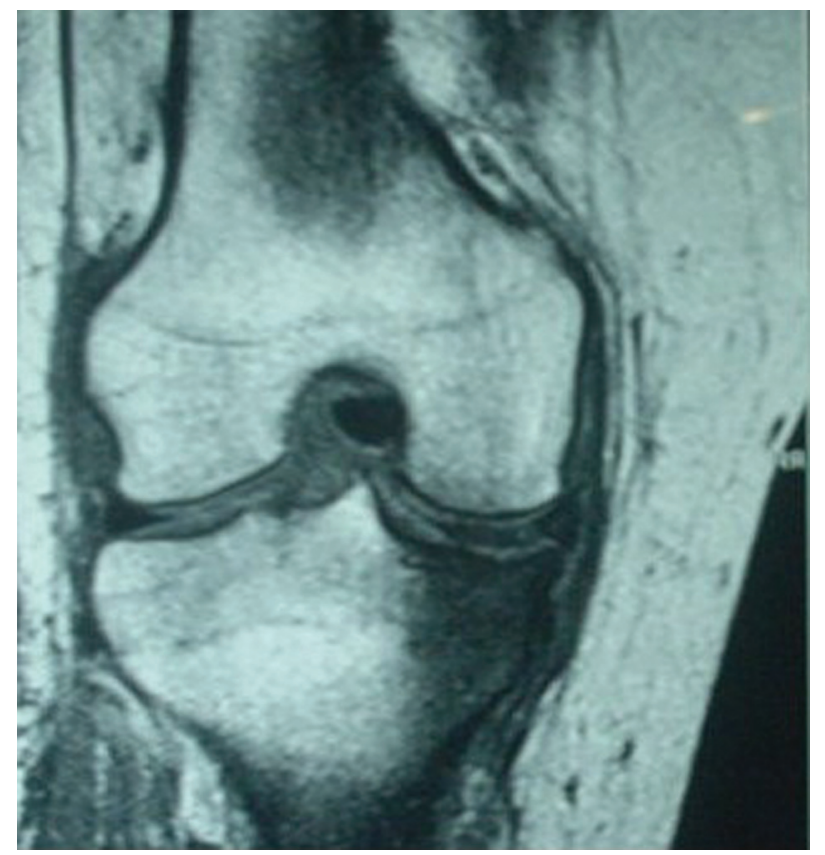

Fig. 3 Imagem de ressonância magnética(RM) com edema ósseo na tíbia, demonstrando lesão por fadiga. 
rotação interna. $\mathrm{O}$ paciente aponta dores mediais na região da inserção dos músculos flexores mediais, a chamada pata de ganso, e na interlinha medial.

Estas dores, provocadas pelo aumento de apoio concentrado na região medial, decorrente do varo e da rotação interna, são chamadas de "bursite da pata de ganso."A palpação demonstra um aumento de sensibilidade nesta região medial. Há uma dificuldade à extensão, que, em alguns casos, provoca dor. A flexão com apoio monopodálico provoca dor. A queixa de dor em repouso é característica do quadro, especialmente durante a noite. $\mathrm{O}$ relato de derrame articular é raro.

\section{Exames por Imagem}

As radiografias demonstram a progressão do varo e o grau de anteriorização da tíbia em relação ao fêmur.

Nas fases iniciais, as alterações radiológicas são muito pouco acentuadas; mas a radiologia é a melhor maneira de avaliar o desvio em varo. A ressonância magnética demonstra o aparecimento de edema ósseo subcondral e da lesão meniscal típica, na transição entre o corpo e o corno posterior do menisco medial (-Figura 3). Em alguns raros casos, a lesão meniscal ocorre na inserção do menisco na raiz do planalto tibial. A avaliação do comprometimento da cartilagem é mais bem feita pela RM, embora não seja característica.

\section{Tratamento}

O tratamento depende muito da fase de evolução da artrose medial. Nas fases iniciais, sem lesão meniscal, o reequilíbrio muscular com exercícios de alongamento e recuperação do quadríceps traz bons resultados. Em casos de dor aguda e imagem de edema ósseo, é interessante a retirada parcial da carga com um par de muletas. Quando a RM detecta lesão meniscal, sem lesão osteocondral detectável, a meniscectomia isolada, seguida de um programa de reequilíbrio da musculatura, traz bons resultados em $90 \%$ dos casos.

É muito importante diferenciar a lesão por fadiga da lesão degenerativa; esta diferenciação é feita pelo estudo radiográfico. Havendo sinais de artrose, estamos diante de uma lesão degenerativa. Neste caso, a meniscectomia não é uma boa indicação, pois leva a bons resultados em torno de $50 \%$ dos casos. $^{3}$

Estudamos 87 doentes portadores de lesão meniscal por fadiga, sem lesão de cartilagem detectável. ${ }^{7,8}$

Todos os pacientes do presente trabalho fizeram RM e radiografias que foram eficientes para o diagnóstico de ausência de lesões na cartilagem, pois em apenas um caso verificamos sinais de lesão condral na artroscopia que não havia sido diagnosticada na avaliação por imagem.

Os nossos pacientes tinham lesão meniscal radial na transição entre o corpo e o corno do posterior do menisco medial diagnosticada pela RM, que foi confirmada em todos os casos pela artroscopia.

Fizemos meniscectomia parcial em todos os casos, procurando retificar a curva meniscal, e excluindo a lesão radial. Embora haja alguma controvérsia quanto à evolução da meniscectomia parcial versus a total, para nós é claro que a meniscectomia parcial traz melhores resultados. Alguns autores $^{9}$ sugerem que, independente do tipo de lesão meniscal, o tratamento conservador pode dar resultados em mais de $50 \%$ dos casos. Acreditamos que esta interpretação pode ocorrer em curtos períodos de avaliação, pois os sintomas da lesão meniscal são incapacitantes em longo prazo.

Os nossos resultados foram considerados bons em $85 \%$ dos casos, com uma média de 87 na escala deLysholm, e os maus resultados se manifestaram logo nos primeiros 6 meses de evolução. Foram 12 pacientes que evoluíram com dor e rapidamente para um quadro de artrose radiológica ou fratura por insuficiência.

Alguns pacientes persistiram com sintomas por aproximadamente dois meses de pós-operatório, mas depois ficaram bem até o final do período de avaliação. A principal queixa destes pacientes era dor ao apoio e dificuldade em subir e descer escadas. Acreditamos que a lesão óssea por fadiga, embora sem tradução radiológica, aliada à fragilidade do quadríceps foi responsável por estes sintomas, pois eles melhoraram com a evolução e a melhora da força do quadríceps.

No que se refere ao percentual alto de bons resultados (85\%), acreditamos que a ausência de lesão da cartilagem seja a responsável.

Higuchi et al $^{10}$ demonstraram que não é a idade e o sexo que são responsáveis pelos maus resultados da meniscectomia, mas a presença de lesão condral. Schimmer et al $^{11}$ observaram bons resultados em $90 \%$ dos pacientes submetidos a meniscectomia com idade e sexo semelhantes aos do nosso grupo, porém sem lesão condral em 12 anos de seguimento.

Em nove pacientes, tivemos um resultado considerado ruim por aparecimento de dor e degeneração articular. Esta degeneração precoce pode ser atribuída ao tipo de lesão meniscal, que, uma vez corrigida, desprotege o planalto tibial.

Em apenas um caso encontramos lesão de cartilagem prévia na avaliação artroscópica, que pode ser relacionada à evolução para artrose; portanto, nos oito casos restantes, houve degeneração articular após a meniscectomia.

Tivemos entre nossos resultados ruins três casos de lesão óssea por insuficiência, chamada também de osteonecrose primária. $^{5}$

Uma vez constatada a lesão condral profunda ou desvio de eixo em varo, comparado com o outro lado, a meniscectomia já não trará resultados satisfatórios. ${ }^{12}$

Desde que estejamos diante de uma artrose estável, segundo os critérios de Ahlbäck, ${ }^{2}$ a osteotomia valgizante da tíbia é uma boa conduta em pacientes em bom estado de saúde.

Preferimos a técnica de Puddu, por apresentar correções previsíveis.

Nos pacientes em estado de saúde comprometido ou idosos funcionais, a prótese unicompartimental é a melhor indicação em portadores de artrose medial com joelhos estáveis, segundo os critérios de estabilidade estabelecidos por Ahlbäck citado em Camanho et al. ${ }^{13}$

Nos pacientes portadores de artrose medial em joelhos com a falência do LCA, ou seja, varos instáveis, a indicação terapêutica é a artroplastia total do joelho (-Tabela 1). 
Tabela 1 Sugerimos o seguinte algoritmo para tratamento

\begin{tabular}{|l|l|}
\hline \multicolumn{2}{|c|}{ Artrose Medial } \\
\hline $\begin{array}{l}\text { Sem Artrose Radiológica } \\
\text { Sem Varismo Patológico } \\
\text { (Ahlback) }\end{array}$ & $\begin{array}{l}\text { Meniscectomia + Reabilitação } \\
\text { muscular }\end{array}$ \\
\hline $\begin{array}{l}\text { 2 - Lesão Condral } \\
\text { Varismo não } \\
\text { instável (Ahlback) }\end{array}$ & Prótese Unicompartimental \\
\hline $\begin{array}{l}3 \text { - Varismo Grave } \\
\text { ou sinais de } \\
\text { instabilidade (Ahlback) }\end{array}$ & Artroplastia total do Joelho \\
\hline
\end{tabular}

\section{Considerações Finais}

Acreditamos que a artrose medial do joelho é um processo evolutivo decorrente de um desequilíbrio muscular, que pode ser tratado a qualquer momento. Quando a indicação terapêutica é adequada e precoce, a expectativa é de bons resultados.

Conflito de Interesses

O autor declara não haver conflito de interesses.

\section{Referências}

1 Molina RD, Sado JJ, Mendlovitz PS, Camanho GL. Avaliação da deformidade rotacional do joelho em paciente com genuvaro e osteoartrose. Rev Bras Ortop 2002;37:430-435

2 Ahlbäck S. Osteoarthrosis of the knee. A radiographic investigation. Acta RadiolDiagn (Stockh) 1968;277(Suppl.):277, 7-72
3 Camanho GL, Hernandez AJ, Bitar AC, Demange MK, Camanho LF. Results of meniscectomy for treatment of isolated meniscal injuries: correlation between results and etiology of injury. Clinics (São Paulo) 2006;61(02):133-138

4 Camanho GL. Dor aguda no joelho do paciente idoso. Rev Bras Ortop 2008;43(09):361-366

5 Norman A, Baker ND. Spontaneous osteonecrosis of the knee and medial meniscal tears. Radiology 1978;129(03):653-656

6 Yamamoto T, Bullough PG. Spontaneous osteonecrosis of the knee: the result of subchondral insufficiency fracture. J Bone Joint Surg Am 2000;82(06):858-866

7 Demange MK, Gobbi RG, Camanho GL. "Fatigue meniscal tears": a description of the lesion and the results of arthroscopic partial meniscectomy. Int Orthop 2016;40(02):399-405

8 Camanho GL. Lesão meniscal por fadiga. Acta Ortop Bras 2009;17 (01):31-34

9 Englund M, Guermazi A, Roemer FW, et al. Meniscal tear in knees without surgery and the development of radiographic osteoarthritis among middle-aged and elderly persons: The Multicenter Osteoarthritis Study. Arthritis Rheum 2009;60 (03):831-839

10 Higuchi H, Kimura M, Shirakura K, Terauchi M, Takagishi K. Factors affecting long-term results after arthroscopic partial meniscectomy. Clin Orthop Relat Res 2000;(377):161-168

11 Schimmer RC, Brülhart KB, Duff C, Glinz W. Arthroscopic partial meniscectomy: a 12-year follow-up and two-step evaluation of the long-term course. Arthroscopy 1998;14(02):136-142

12 Badlani JT, Borrero C, Golla S, Harner CD, Irrgang JJ. The effects of meniscus injury on the development of knee osteoarthritis: data from the osteoarthritis initiative. Am J Sports Med 2013;41(06): 1238-1244

13 Camanho GL, Viegas AC, Camanho LF, Camanho CR, Forgas A. Artroplastiaunicompartimental no tratamento da artrose medial do joelho. Rev Bras Ortop 2007;42(09):285-289 